\title{
Über das Gleichgewicht bei der Schwefelkohlenstoffbildung.
}

\author{
Von \\ F. KOREF.
}

Mit 5 Figuren im Text.

\section{Einleitung.}

Es ist seit langem bekannt, dafs bei der Bildung und bei der Zersetzung des Schwefelkohlenstoffs ein Gleichgewicht vorliegt:

$$
\mathrm{C}+2 \mathrm{~S} \rightleftharpoons \mathrm{CS}_{2} \text {. }
$$

BeRTHELOT ${ }^{1}$ zeigte in einem anschaulichen Versuch, dafs bei der gleichen Temperatur (Rotglut) Schwefelkohlenstoff sich sowohl aus den Elementen bildet - beim Leiten von Schwefeldampf über Kohle, als auch sich zersetzt - beim Leiten von Schwefelkohlenstoffdampf durch eine leere Röhre. Die gleiche Beobachtung machte STEIN. ${ }^{2}$

Aus der Verbrennungswärme, die von Favre und Silbermand, ${ }^{3}$ später von Berthelot ${ }^{4}$ und genauer von ThoMSEN ${ }^{5}$ gemessen wurde, ergab sich die auffallende Tatsache, dals Schwefelkohlenstoff trotz seiner Eigenschaften eines exothermen Körpers aus seinen Elementen unter Wärmeabsorption entsteht. Es ist nach THomsen

$$
\mathrm{C}_{\text {amorph }}+2 \mathrm{~S}_{\text {fest }}=\mathrm{CS}_{2}-26000 \mathrm{cal} \text {. }
$$

Der Schwefelkohlenstoff steht damit zunächst scheinbar im Gegensatz zu den analog zusammengesetzten Kohlenstoffverbindungen:

$$
\begin{aligned}
& \mathrm{C}_{\text {amorph }}+\mathrm{O}_{2}=\mathrm{CO}_{2}+96960 \mathrm{cal} \\
& \mathrm{C}_{\text {amorph }}+4 \mathrm{Cl}=\mathrm{CCl}_{4}+18300 \mathrm{cal} .
\end{aligned}
$$

ThомSEN ${ }^{6}$ fand die richtige Erklärung darin, dafs die negative Verbindungswärme des Schwefelkohlenstoffs ,,als eine Folge der Überführung des Schwefels in den gasförmigen Zustand zu betrachten ist".

Aufgabe der vorliegenden Untersuchungen war es, das interessante Gleichgewicht bei der Bildung des Schwefelkohlenstoffs aus

1 Compt. rend. 67 (1869), 1251.

2 Journ. prakt. Chem. 106 (1869), 316.

3 Ann. Chem. Phys. 34 (1852), 450.

- Ann. Chem. Phys. [5] 23 (1881), 209.

${ }_{5}^{5}$ Th. U. II, 411. Ber. deutsch. chem. Ges. 16 (1883), 2616.

- Th. U. II, 378. 
fester Kohle und Schwefeldampf bei verschiedenen Temperaturen zu bestimmen. Es war zu erwarten, dafs die Kenntnis dieses Gleichgewichts vollständige Klarheit über die thermochemischen Verhältnisse des Schwefelkohlenstoffs bringen würde.

\section{Versuchsmethode und Vorversuche.}

Die Bestimmung des Schwefelkohlenstoffgleichgewichts geschah nach der Strömungsmethode. ${ }^{1}$ Es wurden Versuche über die Zersetzung und über die Bildung des Schwefelkohlenstoffs ausgeführt. Zu diesem Zweck wurde einmal Schwefelkohlenstoff vermittels eines indifferenten Gases (Stickstoffs) durch eine erhitzte Röhre aus Porzellan geleitet; das andere Mal ebenso Schwefeldampf durch eine mit Kohle beschickte Röhre.

Durch orientierende Messungen wurde festgestellt, dafs ziemlich hohe Temperaturen angewendet werden mufsten. Für die Zersetzung des Schwefelkohlenstoffs ist unter $800^{\circ}$ die Reaktionsgeschwindigkeit so klein, dals das Gleichgewicht schwer zu erreichen ist. Auch ist dann der Zersetzungsgrad sehr gering. Die Bildung des Schwefelkohlenstoffs ist eine noch träger verlaufende Reaktion. Infolgedessen wurde von dieser Seite das Gleichgewich erst bei $1000^{\circ}$ erreicht.

Die hohe Versuchstemperatur brachte den Vorteil mit sicb, dafs bezüglich des Schwefelmoleküls vollkommen definierte Verhältnisse vorlagen. Es ist durch verschiedene Messungen aufser $Z$ weifel gesetzt, dafs in dem Temperaturgebiet von $800-1700^{\circ}$ der Schwefeldampf zweiatomig ist.

Für die Dichte des Schwefeldampfes fanden Devilue und Troost ${ }^{2}$ nach der Methode von Dumas (Atmosphärendruck)

$$
\text { für } 860^{\circ} \quad 2.23 \text { und } 1040^{\circ} 2.23 \text {, }
$$

während dem Molekül $\mathrm{S}_{2}$ die Dichte 2.216 entspricht.

V. und C. MExER, ${ }^{3}$ welche nach der Luftverdrängungsmethode arbeiteten, bestimmten die Dichte des Schwefels bei

$$
1560^{\circ} \text { zu 2.17, }
$$

während H. BILTz und V. Meyer ${ }^{4}$ nach der gleichen Methode bei $1719^{\circ} 2.198$ fanden.

Die Messungen von Binfau, ${ }^{5}$ die allerdings viel weniger genau sind, bestätigen diese Resultate.

1 Vgl. Nernst, Theoret. Chemie, 5. Aufl., S. 674.

2 Ann. Chim. Phys. [3] 58 (1860), 257.

B Ber. 12 (1879), 1115.

4 Zeitschr. phys. Chem. 4 (1889), 266.

"Compt. rend. 49 (1859), 799 und Ann. Chem. Phys. [3] 59 (1860), 456. 
Die Ühereinstimmung der nảch der Luftverdrängungsmethode gewonnenen Zahlen mit den nach der Dumasschen Methode erhaltenen beweist, dals in diesem Temperaturintervall eine Dissoziation nicht stattfindet.

Die weitere Spaltung von $\mathrm{S}_{2}$ in die Atome findet erst bei höherer Temperatur, gegen $2000^{\circ}$, statt, ${ }^{2}$ oder möglicherweise bei ganz kleinen Drucken. ${ }^{1}$

Die Bildung und Zersetzung des Schwefelkohlenstoffs verläuft also nach der Gleichung

$$
\mathrm{C}+\mathrm{S}_{2} \rightleftharpoons \mathrm{CS}_{2}
$$

Wir haben ein heterogenes unvollständiges Gleichgewicht; die Reaktion verläuft ohne Volumänderung.

Die Kohle wirkt als Bodenkörper. Die Gleichgewichtskonstante wird also $\mathrm{K}=\frac{\left[\mathrm{S}_{2}\right]}{\left[\mathrm{CS}_{2}\right]}$, wobei $\left[\mathrm{S}_{2}\right]$ und $\left[\mathrm{CS}_{2}\right]$ Konzentrationen in Molen bedeuten. Zur Bestimmung ron $\mathrm{K}$ war es somit erforderlich, die Menge des aus dem Reaktionsgefäls ausgetretenen Schwefels und Schwefelkohlenstoffs zu messen. Beide wurden durch Abkühlung aufgefangen und gravimetrisch bestimmt. - Die Strömungsgeschwindigkeit wurde stark variiert. Die Erreichung des Gleichgewichts wurde daran erkannt, dals mit wechselnder Strömungsgeschwindigkeit $\frac{\left[\mathrm{S}_{2}\right]}{\left[\mathrm{CS}_{2}\right]}$ konstant blieb.

Es war vorauszusehen, dafs für den vorliegenden Fall die Strömungsmethode besonders geeignet war. Wie die Vorversuche ergaben, wächst $\mathrm{K}$ mit steigender Temperatur, d. h. die Dissoziation nimmt zu. Bei abnehmender Temperatur wächst die Konzentration des Schwefelkohlenstoffs. Das Temperaturgefälle kann also auf die Zusammensetzung der aus dem Reaktionsgefäls ausströmenden Gase keinen Einfluls ausüben, wenn dieser Teil des Rohres frei von Kohle, eine Bildung von Schwefelkohlenstoff, infolgedessen unmöglich ist. (Diese Erwartung erfüllte sich nur bei den Versuchen über Bildung von Schwefelkohlenstoff, bei Zersetzungsversuchen flog ein Teil der entstehenden Kohle als feiner Staub mit; hierüber s. w. u.)

Was nun die Modifikation des bei der Zersetzung sich aus-

1 Nernst, Z. f. Eleltrochem. 9 (1903), 626. - v. Wartenbera, Z. anorg. Chem. 56 (1907), 320 .

" Pleuner, Zeitschr. phys. Chem. 68 (1909), 147. 
scheidenden Kohlenstoffs anlarigt, so berichtet BerTHELOT, ${ }^{1}$ die Kohle habe sich in kleinen Blättchen von metallischem Glanz abgesetzt, welche viel Graphit enthielten. Einen Beweis für das Vorhandensein von Graphit gibt Berthelot nicht an. Bei der Untersuchung der in den Vorversuchen an den Wandungen des Porzellanrohres abgeschiedenen Kohle hat sich BerThELots Mitteilung nicht bestätigt, die Anwesenheit von Graphit konnte nicht nachgewiesen werden. - Die Kohle haftete fest am Porzellanscherben, konnte mechanisch nicht entfernt werden; sie verbrannte erst nach längerem Erhitzen im Sauerstoffgebläse.

Herr Dr. v. Wartenberg besafs die Freundlichkeit, die Untersuchung auf Graphit vorzunehmen. Die Kohle wurde auf chemischen Wege von der Porzellanmasse getrennt. Einige Bruchstücke des Porzellanrohres wurden mit Flufssäure und Schwefelsäure wiederholt abgeraucht, der Rückstand mit Wasser aufgeschlämmt, zur Lösung von Eisenoxyd (aus der Porzellanmasse) mit Salzsäure behandelt, wieder geschlämmt und abgeraucht, bis die Kohle allein zurückblieb. Diese wurde dann trocken mit Kaliumchlorat verrieben, mit Schwefelsäure versetzt und auf dem Wasserbad auf $60^{\circ}$ erwärmt. Nach einer halben Stunde war alles gelöst, die Lösung war klar und farblos. Die Kohle war also restlos zu Kohlensäure oxydiert worden. Bei Anwesenheit von Graphit hätte sich Graphit. säure bilden müssen.

Bei der Schwierigkeit, amorphe Kohle rein zu erhalten, ${ }^{2}$ wurde zur Füllung des Reaktionsgefäfses Achesongraphit benutzt (Amerik. Pat. Nr. 711 281), der von flüchtigen Verunreinigungen vollkommen frei ist. ${ }^{3}$ Es wurden aber zuerst zahlreiche Versuche über die Zersetzung des Schwefelkohlenstoffs vorgenommen, so dafs der Graphit mit einer dünnen Schicht des hierbei freiwerdenden Kohlenstoffs überzogen war, als mit Versuchen von der Gegenseite begonnen wurde. Ein Unterschied zwischen den Gleichgewichtskonstanten bei der Bildung und Zersetzung war nicht wahrzunehmen.

\section{Versuchsanordnung.}

Der bei den Versuchen als indifferentes Gas benutzte Stickst off wurde einer Stickstoffbombe entnommen. Da dieser nicht rein war, vor allem erhebliche Mengen Sauerstoff enthielt, mulste er

1 Ann. Chim. Phys. [4] 19 (1870), 423 und [5] 23 (1881), 209.

2 Vgl. Jos', Dissertation 1908, S. $11 \mathrm{ff}$.

${ }^{3} \mathrm{Vgl}$. Haber, Z. f. Elektrochem. 9, 379. 
sorgfältigst gereinigt werden. Er wurde zu diesem Zwecke zu. nächst in einen $15 \mathrm{l}$ fassenden aus Glasflaschen zusammengesetzten Gasometer eingefüllt, in welchem einige Stangen gelben Phosphors in einem Körbchen aus Kupferdraht aufgehängt waren. Der Phosphor absorbierte den Sauerstoff fast vollständig. - Der Stickstoff passierte eine mit Kalilauge gefüllte Schlangenwaschflasche, dann ein langes Chlorcalciumrohr, ein mit Kupferoxyd ge. fülltes glühendes Porzellanrohr zur Verbrennung von spurenweise vorhandenem Wasserstoff und im Gasometer gebildetem Phosphorwasserstoff, eine Schlangenwaschflasche mit konzentrierter Schwefelsäure, strich dann zur Absorption der letzten Reste von Sauerstoff über eine glühende Kupferspirale und schliefslich zur vollkommenen Trocknung durch ein $25 \mathrm{~cm}$ langes Phosphorpentoxydrohr.

Das Variieren der Strömungsgeschwindigkeit erfolgte vermittels eines mit Quecksilber abgedichteten Schraubenregulierhahnes aus Eisen.

Der in der beschriebenen Weise gereinigte Stickstoff trat nun in die Gefälse, in denen er sich mit Schwefelkohlenstoff- und Schwefeldampf belud. Diese waren aus Glas hergestellt, ihre Gestalt ist aus der Fig. 1 ersichtlich. Der Stickstoff perlte durch den Schwefelkohlenstoff im Gefäls $A$. Zur Vermeidung gröfserer Temperaturschwankungen befand sich das Schwefelkohlenstoffgefäls in einem

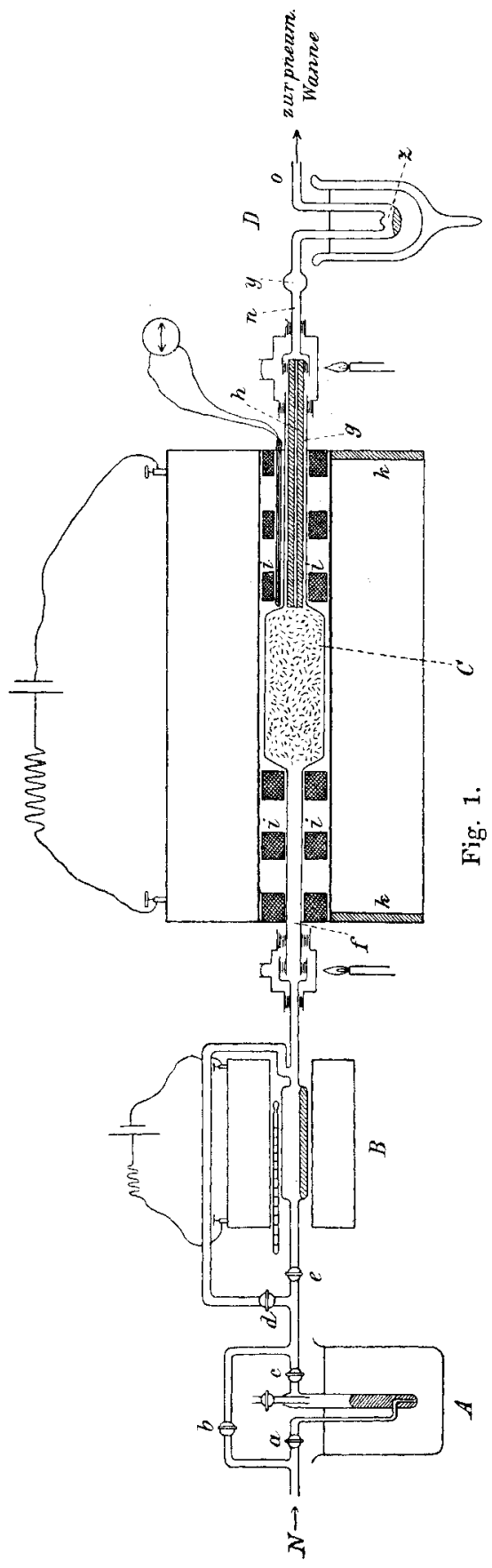


grörseren Becherglas, das mit Wasser von Zimmertemperatur gefüllt war. Der Dampfdruck des Schwefelkohlenstoffs beträgt rund $300 \mathrm{~mm}$ $\mathrm{Hg}$ bei $20^{\circ}$. 11 Stickstoff nimmt dann, Sättigung vorausgesetzt, $1.34 \mathrm{~g} \mathrm{CS}_{2}$ mit. Der Stickstoff konnte durch eine Rohrabzweigung auch um das Schwefelkohlenstoffgefäls herumgeleitet werden.

Das Schwefelgefäls $B$ bestand aus einem $15 \mathrm{~cm}$ langen, $2 \mathrm{~cm}$ weiten Glasrohr. Der Stickstoff strich über den geschmolzenen Schwefel. Die Heizung erfolgte durch einen elektrischen Ofen. Dieser bestand aus einer mit Nickeldrabt umwickelten Kupferröhre, die in einem mit gebrannter Magnesia gefüllten Asbestkasten safs. Durch vorgelegte Widerstände wurde die Temperatur des Ofens zwischen 260 und $320^{\circ}$ variiert, je nachdem die Versuche es erforderten. Die Temperatur wurde an einem Quecksilberthermometer abgelesen. - Auch das Schwefelgefäls war mit einer Rohrabzweigung versehen, die es gestattete, den Stickstoff um das Gefäls herumzuleiten. Durch geeignete Stellung der Hähne $a, b, e$ und $d, e$ konnte der Stickstoff also entweder nur mit Schwefelkohlenstoff oder nur mit Schwefeldampf oder mit einer Mischung von beiden beladen, schlielslich auch unbeladen in das Reaktionsgefäls eintreten. Das Mischungsverbältnis konnte durch Änderung der Temperaturen variiert werden.

Das Reaktionsgefä $f$ s bestand aus einer $62 \mathrm{~cm}$ langen, in der Mitte ballonartig erweiterten aufsen glasierten Porzellanröhre, welche in der Königlichen Porzellanmanufaktur hergestellt wurde. Die ballonartige Erweiterung, der eigentliche Reaktionsraum, $15 \mathrm{~cm}$ lang, war mit Körnern aus Achesongraphit gefüllt, welche durch Zerstolsen im Mörser und Aussieben auf die Korngröfse von 1-2 mm gebracht waren. Da verschiedene Porzellanrohre benutzt wurden, deren Volumen nicht gleich war, wurde jedesmal der Inhalt des Ballons, bevor die Röhre in Gebrauch genommen wurde, durch Auswägen mit Wasser unter Abrechnung der zylindrischen engen Rohrteile $f$ und $g$ bestimmt. Die Graphitmenge wurde ebenfalls gewogen, so dafs das verbleibende Leervolumen des Ballons berechnet werden konnte (spez. Gew. des Graphits 2.25). Das Zuführrohr $f$ war zum grölsten Teil seines Querschnittes durch einen eingelegten Porzellanstab ausgefüllt (in der Figur weggelassen). Infolgedessen war hier die Strömungsgeschwindigkeit besonders grofs, ein Zurückdestillieren des Schwefels und Schwefelkohlenstoffs daher auch bei langsamer Strömung nicht möglich.

In dem Abführrohr $g$, das $7 \mathrm{~mm}$ weit war, lag eine Porzellancapillare $h$. Diese war in weiter unten zu beschreibenden Weise 
derart in dem Porzellanrohr befestigt und abgedichtet, dafs die Gase gezwungen wurden, durch sie hindurch das Reaktionsgefäfs zu verlassen. Diese Anordnung, die den Zweck hatte, die Capillare auswechseln und zeitweise durch eine Quarzcapillare ersetzen zu können, erwies sich im Verlauf der Versuche als erforderlich (siehe weiter unten). Die Weite der verschiedenen Capillaren betrug $1-2.5 \mathrm{~mm}$.

Das Reaktionsgefäls safs, durch breite Chamotteringe $i i$ getragen, in einem $45 \mathrm{~cm}$ langen elektrischen Ofen. Dessen Heizröhre wurde von einer mit Platinband umwickelten Röhre aus Marquardtmasse von der Königlichen Porzellanmanufaktur gebildet. Die Heizröhre war vermittels Stützen aus Magnesiaplatten $k k$ in einen mit gebrannter Magnesia gefüllten Asbestkasten montiert, welcher zur Verminderung der Wärmeabgabe durch Strahlung in einem Kasten aus blankem Aluminiumblech sals. Der Heizstrom wurde der städtischen Leitung entnommen, die Temperatur wurde durch vorgelegte Widerstände variiert und reguliert. Die Schwankungen betrugen nicht mehr als $\pm 3^{0}$.

Die Temperaturmessung geschah durch ein Thermoelement $m$ $(\mathrm{Pt}-\mathrm{PtRh})$, das mit Hilfe des Goldschmelzpunktes geeicht wurde $\left(1064^{\circ}=10.21\right.$ Millivolt). Die Spannung des Thermoelementes wurde auf einem genauen Millivoltmeter abgelesen. Die Temperaturen wurden auf Grund der Angaben von HoLBonN und DAY ${ }^{1}$ aus den Spannungen ermittelt. Das Thermoelement lag aufserhalb des Reaktionsgefälses. Die Temperaturdifferenz zwischen aufsen und innen wurde $\mathrm{zu}$ rund $8^{\circ}$ gefunden und berücksichtigt.

An das Reaktionsgefäls schlofs sich dann eine Glasröhre $n$ an, die zu den Auffangegefälsen führte. Dieses Verbindungsstück mufste ebenso wie die Verbindung zwischen dem Schwefelgefäls $B$ und dem Porzellanrohr samt den aus dem Ofen herausragenden Enden des Porzellanrohres auf einer Temperatur von $350-400^{\circ}$ gehalten werden, um Kondensation des Schwefels zu vermeiden.

Die Verbindung und Dichtung ron Glas- und Porzellanrobr machte deshalb erhebliche Schwierigkeiten. Nach verschiedenen Versuchen erwies sich folgende, der üblichen Quecksilberdichtung bei Schliffen nachgebildete Anordnung als am einfachsten und zweckmälsigsten (siehe Fig. 2, in gröfserem Mafsstab als in der Hauptfigur). Das Ende des Glasrohres war zu einer $1.5 \mathrm{~cm}$ langen Kappe $p$ erweitert, deren lichte Weite etwa $1 \mathrm{~mm}$ mehr betrug, als

1 Drudes Ann. 2 (1900), 526. 
der äufsere Durchmesser des Porzellanrohres. Die Kappe wurde über das Porzellanrohr geschoben, der Zwischenraum zwischen beiden sorgfältig mit Asbest vollgestopft. Über die Ansatzstelle war nun ein kleines Eisenrohr $r$ geschoben, das der Weite des Porzellanrohres entsprechend an seinen Enden verengt war. Hier wurde das

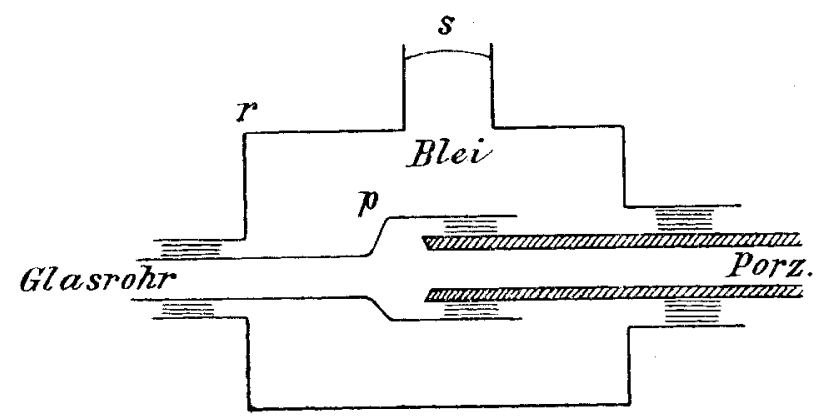

Fig. 2 a.

Eisenrohr ebenfalls durch Stopfen mit Asbest am Glas- und Porzellanrohr befestigt. Der so entstehende Hohlraum wurde durch eine oben am Eisenrohr angebrachte Öffnung $s$ gefüllt mit flüssigem Blei.

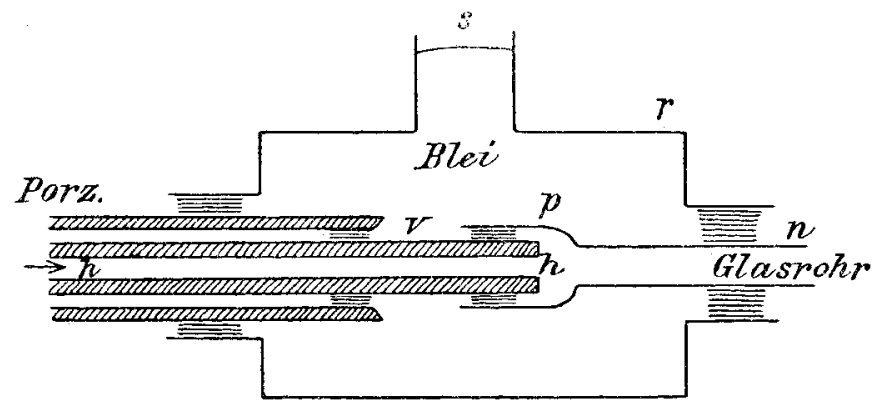

Fig. 2 b.

Durch ein darunter gestelltes Bunsenbrennerflämmchen wurde die ganze Anordnung auf der erforderlichen Temperatur, gegen $400^{\circ}$, gehalten (Schmelzpunkt des Bleis $330^{\circ}$ ). - In der gleichen Weise wurde auch die aus dem Porzellanrohr herausragende Capillare $h$ in dem Porzellanrohr befestigt bei $v$ (Fig. 2b). Die hier angebrachte Bleidichtung verschlofs gleichzeitig auch den Ansatz des Glasrohres $n$ an die Capillare $h$. Man sieht aus der Figur, dals die Gase so gezwungen waren, beim Ausströmen aus dem Reaktionsraum den Weg durch die Capillare zu nehmen. ${ }^{1}$

1 Ein anderes Verfahren, eine gasdichte Verbindung, welche Erhitzen verträgt, zwischen Glas und Porzellan herzustellen, sei hier noch erwähnt, da 
Als Auffangegefäfs für den ausströmenden Schwefel- und Schwefelkohlenstoffdampf diente ein $\mathbf{U}$-Rohr $D$, welches durch in einem Dewargefäfs befindliche feste Kohlensäure und Alkohol abgekühlt wurde. Oben an dem U-Rohr war eine Kugel $y$ angebracht, in der sich der gröfste Teil des Schwefels kondensierte. Unten hatte das U-Rohr eine Erweiterung $\approx$, welche bewirkte, dafs die Gase sich längere Zeit in dem kalten Raum aufhalten mulsten, die Kondensation infolgedessen vollständig wurde. Der Dampfdruck des Schwefelkohlenstoffs bei dem Sublimationspunkt der festen Kohlensäure $\left(-78^{\circ}\right)$ ist kleiner als $1 \mathrm{~mm}$, wie sich durch Extrapolation der Dampfdruckkurve von REGNAULT ${ }^{1}$ ergibt. Da der Partialdruck des Schwefelkohlenstoffs vor der Kondensation ungefähr $300 \mathrm{~mm}$ betrug, so konnte der Versuchsfehler, der durch den Dampfdruck des Schwefelkohlenstoffs bei der Kondensationstemperatur sich ergab, vernachlässigt werden. Er betrug etwa $1 / 3 \%$.

Der Stickstoff wurde schliefslich in einer pneumatischen Wanne aufgefangen und volumetrisch gemessen.

War ein Versuch beendigt, so wurde der Stickstoffstrom abgestellt, das Auffangegefäls $D$ bei $n$ mit einem Handgebläse abgescholzen und auch an der anderen Seite bei o zugeschmolzen, so dafs es gänzlich geschlossen, ohne Verlust an Schwefelkohlenstoff aus der Kühlmasse genommen und auf Zimmertemperatur gebracht werden konnte. Das geschlossene Gefäls wurde dann gewogen $\left(G e f a ̈ l s+C_{2}+S\right)$. Zur Entfernung des Schwefelkohlenstoffs mulste das Gefäls zunächst geöffnet werden. Zu diesem Zweck wurde nahe dem zugeschmolzenen Ende das Glas mit einer kleinen Stichflamme erhitzt, bis es an dieser Stelle weich und durch den innen herrschenden Schwefelkohlenstoffüberdruck aufgeblasen wurde. Dann wurde das Glas etwa $1 \mathrm{~cm}$ vom Ende mit einem scharfen Glasmesser angeritzt und das Endstück abgesprengt. Die ganze Manipulation wurde über einer $50 \mathrm{~cm}$ weiten groĺsen Krystallisierschale, welche

sie für manche Zwecke brauchbar sein dürfte. Das Glasrohr wurde in das Porzellanrohr mit Porzellankitt (Porzellanmehl und Wasserglas) eingekittet, und die Kittung langsam getrocknet. Dann wurde die Kittstelle und die nächsten $11 / 2 \mathrm{~cm}$ vom Glas- und Porzellanrohr mittels Rössuerschen Glanzplatins mit einem ganz dünnen fest haftenden Platinüberzug versehen, auf diesen dann auf elektrolytischem Wege eine $1 / 2 \mathrm{~mm}$ dicke Silberschicht niedergeschlagen. Das Silber sals fest auf Porzellan und Glas und verschlofs die Poren des Kittes. Die Verbindungsstelle konnte mit einer kleinen Bunsenflamme heifs gehalten werden.

1 Mém. l'Acad. sciences 26 (1862), 394.

Z. anorg. Chem. Bd. 66 . 
auf schwarzem Glanzpapier stand, ausgeführt, so dafs abspringende Glassplitterchen mit einem Pinsel gesammelt und nachher mit zur Wägung gebracht werden konnten. Der Schwefelkohlenstoff wurde dann mit einer Wasserstrahlluftpumpe abgesaugt. Darauf wurde wieder gewogen (Gefäls $+S$ ). Nun wurde ebenso wie vorher auch das andere geschlossene Ende abgesprengt, zur Kontrolle wieder gewogen, da kleine Verluste bei der geringen Schwefelmenge sich stärker bemerkbar gemacht hätten. Sodann wurde der im Gefäls

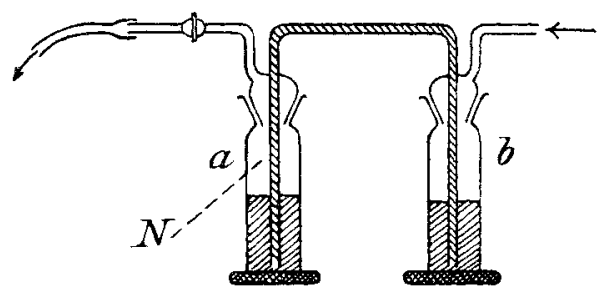

Fig. 3.

sitzende Schwefel unter Durchblasen von Luft und Erhitzen über einem Bunsenbrenner verbrannt, und zum Schlufs das leere Gefäls gewogen. Die Differenz der 1. und 2. Wägung ergab das Gewicht des Schwefelkohlenstoffs, die der 3. und 4. das Gewicht des Schwefels. Die Versuchsdauer wurde so eingerichtet, dals die Gesamtmenge von Schwefelkohlenstoff und Schwefel 0.7-1.5 g betrug.

Sollte sich nach dem Abschmelzen des U-Rohres gleich ein weiterer Versuch anschlielsen, so wurde sofort ein anderes U-Rohr mit dem Handgebläse angesetzt. Hierbei durfte aber nicht in der üblichen Weise in das Gefäls hineingeblasen werden, da sonst Sauerstoff in den Reaktionsraum gekommen wäre, der die Reaktion störend beeinflufst hätte. Dies zu vermeiden, bediente ich mich eines kleinen, aus zwei gegeneinandergesetzten $W$ aschflaschen gebildeten Stickstoffgasometers (Fig. 3), dessen Sperrflüssigkeit konzentrierte Schwefelsäure war. Die mit Stickstoff gefüllte Flasche $a$ wurde mit dem anzusetzenden Gefäls durch einen Gummischlauch verbunden. Durch leichtes Blasen in die andere Flasche $b$ wurde durch die Flüssigkeit hindurch auf das geschlossene Stickstoffrolumen ein Druck ausgeübt, der ausreichte, die Ansatzstelle zu verblasen. Abschmelzen und Ansetzen dauerte etwa 10 Minuten. - Konnte der nächste Versuch nicht sofort angeschlossen werden, so wurde das Reaktionsgefäfs durch einen Stickstoffstrom ausgespült. 


\section{Versuchsergebnisse.}

Bei den Versuchen über die Zersetzung des Schwefelkohlenstoffs ergab sich die eigenartige Tatsache, dafs die Werte für den Zersetzungsgrad allmählich zu fallen begannen, wenn das Reaktionsgefäls eine Zeitlang benutzt war. Das Fallen der Werte begann etwa beim 6 . oder 7. Versuch. Es fiel ferner auf, dafs die Versuche, vor deren Beginn das Reaktionsgefäls mit einigen Litern Stickstoff ausgespült worden war, höhere Werte gaben als diejenigen, die sich unmittelbar an den vorhergehenden anschlossen. Einige Beispiele mögen dies zeigen. Wir bezeichnen mit $x$ den Dissoziationsgrad, $100 x$ ist dann die Zersetzung in Prozenten. Bei $900^{\circ}$ ergab ein Versuch nach vorheriger Ausspülung des Apparates mit 21 Stickstoff die Zersetzung $100 x=8.27$, der sich unmittelbar anschliefsende $100 x=6.51$ bei annähernd der gleichen Strömungsgeschwindigkeit. Der nächste Versuch nach Ausspülung des Apparates ergab $100 x=$ 8.26. Dem Gleichgewicht bei dieser Temperatur, das aus den ersten Versuchen sich ergab, entsprach eine Zersetzung von $100 x=$ 10.3. - Bei $820^{\circ}$ gab ein Versuch nach vorheriger Ausspülung $100 x=4.74$, während sich der daran anschlielsende $100 x=4.09$ lieferte bei gleicher Strömungsgeschwindigkeit. Diese Beobachtung wurde bei zahlreichen Versuchen gemacht. Diese hier anzuführen, hat keinen Zweck, da sie für die Bestimmung des Gleichgewichts wertlos sind, wie sich bald ergab.

Es lag also nahe anzunehmen, dafs während der Versuche sich ein Katalysator bildete, der auf die ausströmenden Gase, mit denen der frei werdende Kohlenstoff in feinverteilter Form wohl mitflog, einwirkte und ein Gebiet des Temperaturgefälles die bei tieferer Temperatur eintretende Gegenreaktion, die Rückbildung von $\mathrm{CS}_{2}$, begünstigte. Eine Erhöhung der Geschwindigkeit der ausströmenden Gase durch Einlegen einer ganz engen ( $1 \mathrm{~mm}$ weiten) Porzellancapillare hatte keinen merklichen Erfolg. Die Porzellancapillare wurde nun durch eine Quarzcapillare ersetzt. Nachdem eine grölsere Menge Schwefelkohlenstoff durch den Apparat geschickt worden war, wurde die Quarzkapillare schnell herausgezogen. Sie war zuerst vollständig klar, dann bildete sich an ihrer Innenwand ein weifser Beschlag, der beim Glühen nicht verschwand, nur in Flufssäure löslich war. Gleichzeitig wurde Geruch nach Schwefelwasserstoff wahrgenommen.

Die Annahme, dals die Kieselsäure des Porzellanrohres vom 
Schwefel oder Schwefelkohlenstoff unter Bildung eines Siliciumsulfids angegriffen wurde, bestätigte sich. Nach Fremy, ${ }^{1}$ der als erster Siliciumsulfid $\mathrm{SiS}_{2}$ darstellte, entsteht dieser Körper bei der Einwirkung von $\mathrm{CS}_{2}$ auf Kieselsäure, eine Reaktion, die durch Anwesenheit von Kohle begünstigt wird. An der feuchten Luft zersetzt sich dieser Körper unter Bildung von $\mathrm{SiO}_{2}$ und $\mathrm{H}_{2} \mathrm{~S}$. (Daher der weifse Beschlag im Porzellanrohr und der $\mathrm{H}_{2} \mathrm{~S}$-Geruch.) FrÉMY hat bereits beobachtet (l. c.), dafs Glas und Porzellan bei starkem Erhitzen unter Einwirkung von Schwefelkohlenstoff $\mathrm{SiS}_{2}$ bilden. ${ }^{2}$

Die allmähliche Bildung von Siliciumsulfid erklärt die beobachteten Anomalien vollkommen.

Vermutlich wirkt das Siliciumsulfid als Schwefelüberträger auf die mitfliegende freigewordene Kohle.

Von den Zersetzungsversuchen wurden daher zur Bestimmung des Gleichgewichtes nur die 5-6 ersten Versuche mit neuem Rohr berücksichtigt.

Bei den Versuchen von der Gegenseite, bei denen Kohle nicht mitfliegen konnte, wurde ein Fallen der Werte nicht beobachtet. Hier konnten also auch die späteren Versuche zur Bestimmung des Gleichgewichtes benutzt werden.

Wir müssen annehmen, dals auch bei diesen Versuchen das anwesende $\mathrm{SiS}_{2}$ als Katalysator wirkt, hier aber die Hauptreaktion katalysiert. Die Reaktionsgeschwindigkeit wird also von der Konzentration des anwesenden Siliciumsulfids beeinflufst, ist also von der Vorbehandlung abhängig. Die ursprüngliche Absicht, die Geschwindigkeit der Reaktion

$$
\mathrm{C}+\mathrm{S}_{2}=\mathrm{CS}_{2}
$$

bei verschiedenen Temperaturen zu bestimmen, diese Untersuchung auf verschiedene Kohlearten auszudehnen, konnte infolgedessen nicht ausgeführt werden.

Die für die Bestimmung des Gleichgewichtes in Betracht kommenden Versuchsergebnisse sind in den folgenden Tabellen 1-4 zusammengestellt. Die gewogenen Mengen $\mathrm{CS}_{2}$ und $\mathrm{S}_{2}$ (Kolumne 2a und 3 a) wurden in ccm bei $T^{0}$ umgerechnet nach dem Ausdruck

$$
g \cdot \frac{22400 T}{M \cdot 273}
$$

I Ann. Chim. Phys. 38 (1853), 314.

2 Vgl, auch Bitrz, Ber. 1908, 3345. 


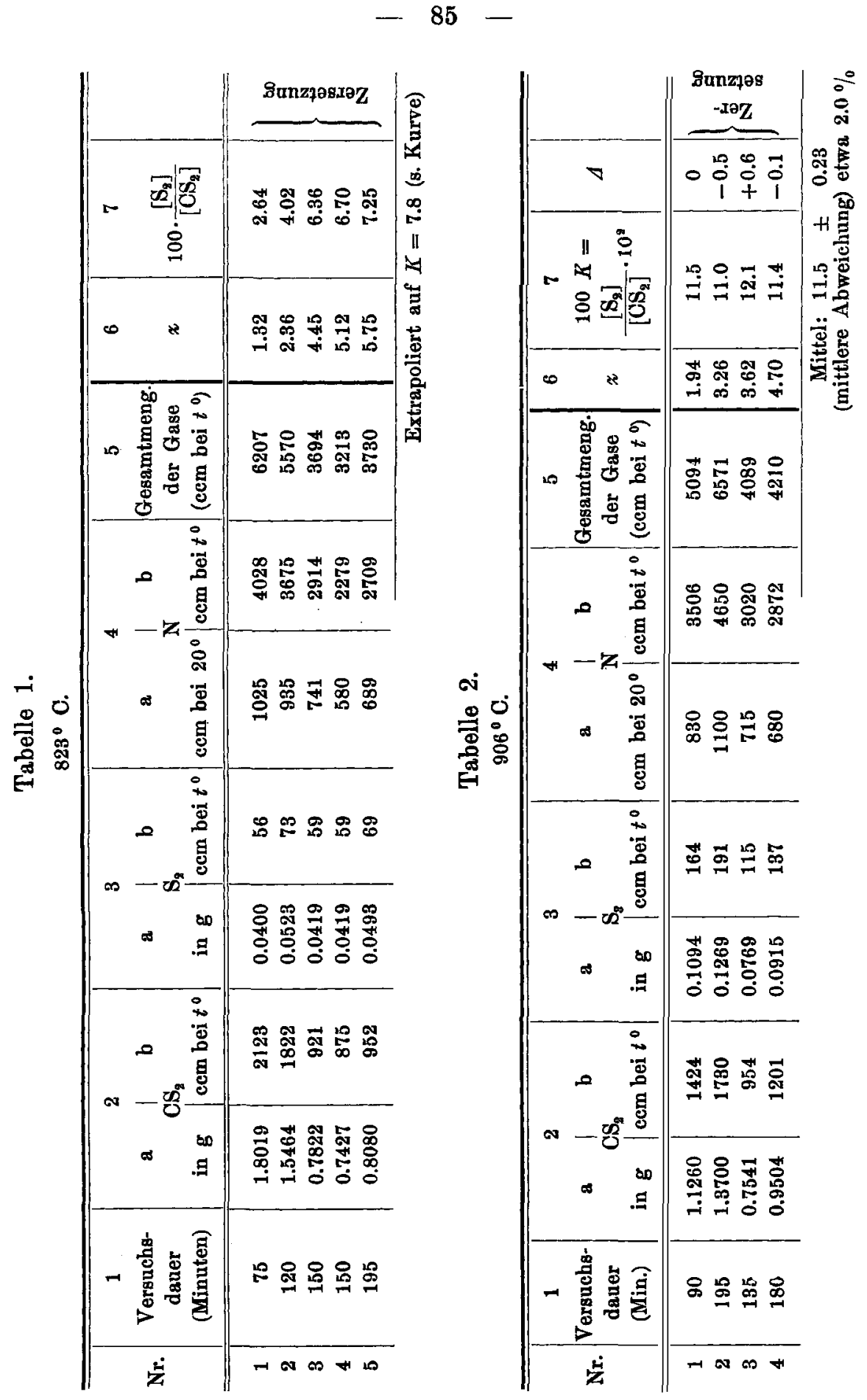



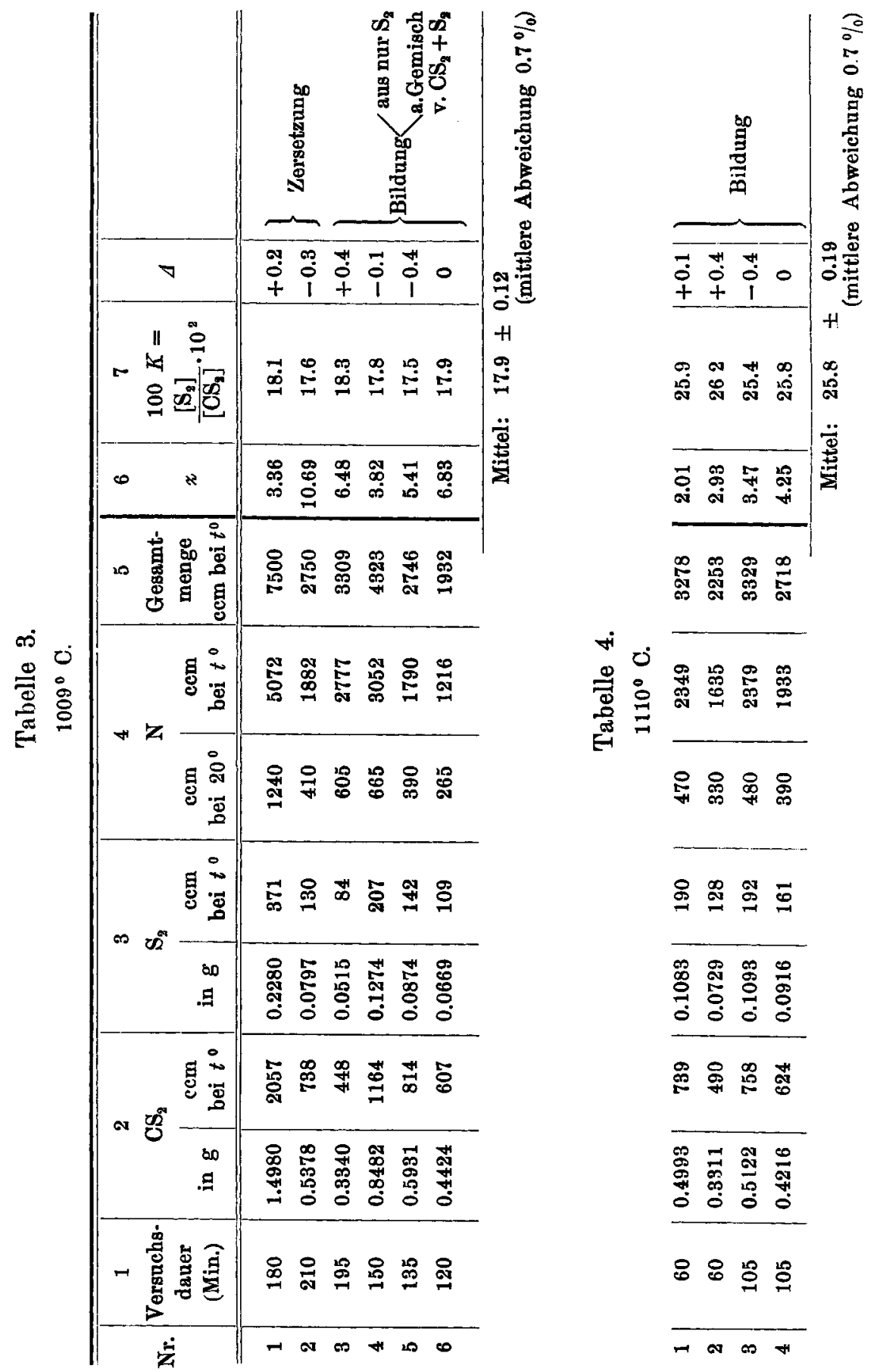

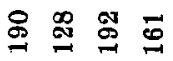

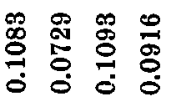

承学怘范

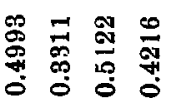

용요용

- a m 
Hierin bedeutet $g$ die gewogene Menge in Grammen, $T$ die absolute Temperatur, $M$ das Molekulargewicht, also $\mathbf{7 6 . 1 2}$ für den Schwefelkohlenstoff, 64.12 für den Schwefel. Das bei Zimmertemperatur gemessene Stickstoffrolumen wurde ebenfalls in $\mathrm{ccm}$ bei $T^{0}$ umgerechnet. Die so gefundenen Gröfsen sind in Kolumne $2 \mathrm{~b}, 3 \mathrm{~b}$ und $4 \mathrm{~b}$ zusammengestellt. Durch die Addition von $2 \mathrm{~b}, 3 \mathrm{~b}$ und $4 \mathrm{~b}$ er-

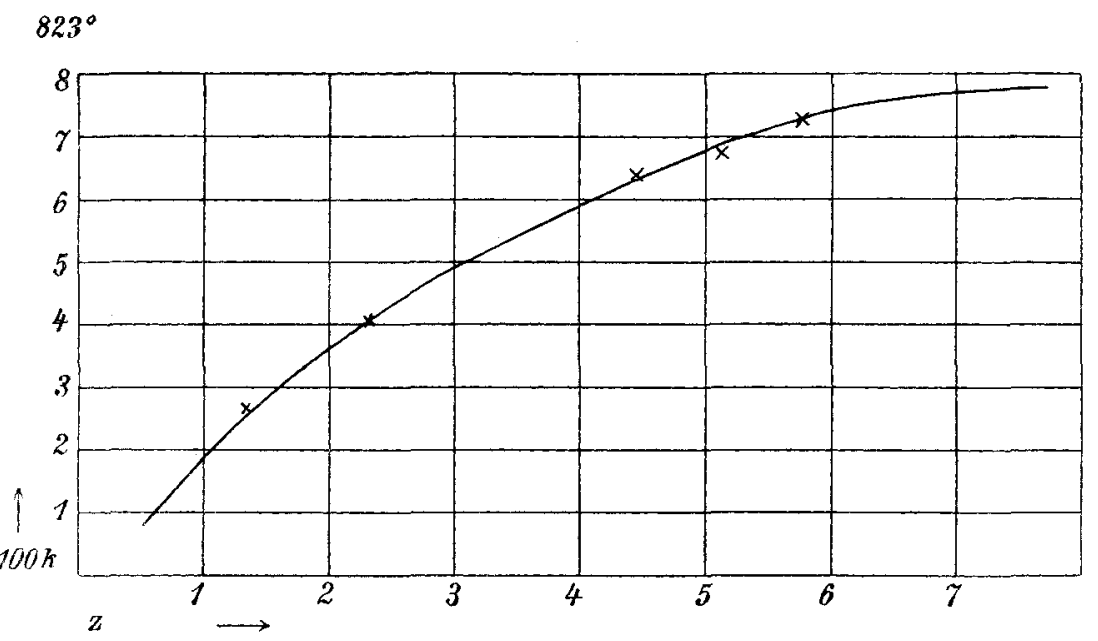

Fig. 4.

gab sich das für die Berechnung der Strömungsgeschwindigkeit wichtige Gesamtvolumen der durchgeschickten Gase, gemessen in ccm bei $T^{0}$ (Kolumne 5).

In Kolumne 6 ist

$$
z=\frac{\text { Versuchsdauer } \times \text { Reaktionsraum }}{\text { Durchgeschickte Gasmenge }},
$$

$\approx$ ist also der reziproke Wert der Strömungsgeschwindigkeit und bedeutet die Zeit (in Minuten), die $1 \mathrm{ccm}$ Gas sich im Reaktionsraum authält. In Kolumne 7 bedeutet $k=\frac{\left[\mathrm{S}_{2}\right]}{\left[\mathrm{CS}_{2}\right]}$ die Gleichgewichtskonstante. $\Delta$ ist die Abweichung vom Mittel.

Bei $823^{\circ}$ (Tabelle 1) wurde das Gleichgewicht nicht erreicht, kann aber extrapoliert werden. $k$ ergibt sich zu 7.8.

Bei $823^{\circ}$ wie bei $906^{\circ}$ (Tabelle 2) liegen nur Versuche über Zersetzung von $\mathrm{CS}_{2}$ vor.

Versuche von beiden Seiten wurden bei $1009^{\circ}$ ausgeführt (Tabelle 3). Nr. 1 und 2 sind Zersetzungsversuche, die übrigen sind 
Versuche über Bildung von $\mathrm{CS}_{2}$, und zwar wurde in Versuch 3 Schwefeldampf über die Kohle geleitet, in Versuch $4-6$ ein Gemisch von Schwefel- und Schwefelkohlenstoffdampf, in welchem der Schwefel in bezug auf das Gleichgewicht im Überschufs war.

Bei $1110^{\circ}$ (Tabelle 4) wurden nur Versuche über Bildung ausgefübrt.

Die Übereinstimmung der Konstanten genügt den Anforderungen, die an die Genauigkeit einer Gleichgewichtskonstanten zu stellen sind. Die mittlere Abweichung, die nach der bekannten Formel $\mathfrak{F}=\sqrt{\frac{\Sigma \Delta^{2}}{n(n-1)}}$ ermittelt wurde, beträgt in Tabelle 2 etwa $2 \%$, in Tabelle $30.7 \%$ in Tabelle $40.6 \%$.

Es wurde versucht, die Gleichgewichtskonstante bei einer noch höheren Temperatur $\left(1200^{\circ}\right)$ zu bestimmen. Die Bildung von Siliciumsulfid wurde dabei aber so stark, dafs das in die Capillare sublimierende $\mathrm{SiS}_{2}$ diese nach kurzer Zeit verstopfte und die Versuche unmöglich machte.

\section{Thermodynamische Berechnung.}

Die Berechnung der Wärmetönung erfolgte nach der Gleichung der Reaktionsisochore

$$
Q=\frac{4.571 \cdot T_{1} \cdot T_{2}\left(\lg k_{2}-\lg k_{1}\right)}{T_{2}-T_{1}}
$$

Nach Tabelle 5 beträgt $Q$ im Mittel 12500 cal.

Tabelle 5.

\begin{tabular}{c|c|c|c|c}
\hline \hline$t^{0}$ & $T$ abs. & $K \cdot 10^{2}$ & $\lg k_{2}-\lg k_{1}$ & $Q$ \\
\hline \hline$<23$ & 1096 & 7.8 & 0.1686 & 12000 \\
$<906$ & 1179 & 11.5 & 0.1922 & 12900 \\
$<1009$ & 1282 & 17.9 & 0.1587 & 12700 \\
1110 & 1383 & 25.8 & \multicolumn{2}{|c}{ Mittel: $12500 \mathrm{cal}$}
\end{tabular}

Die Bildungswärme des Schwefelkohlenstoffs aus fester Kohle und Schwefeldampf ist in der Tat positiv, wie dies Thomsen vorausgesagt hat (vgl. die Einleitung). Wir können den Schwefelkohlenstoff als einen exothermen Körper betrachten. Die von Thomsen aus der Verbrennungswärme ermittelte negative Bildungswärme erklärt sich aus der hohen Verdampfungswärme des Schwefels. 
Vermittels der Wärmetönung $Q=12500$ können wir die Gleichgewichtskonstanten und den Zersetzungsgrad für ein grölseres Tem. peraturgebiet berechnen. Die so gefundenen Werte haben angenäherte Richtigkeit unter der Voraussetzung, dafs $Q$ sich mit der Temperatur wenig ändert. Dies ist tatsächlich der Fall, wie im nächsten Abschnitt gezeigt wird. Bezeichnen wir mit $x$ den Dissoziationsgrad, so besteht zwischen $k$ und $x$ die Beziehung

$$
k=\frac{x}{1-x} \text { oder } x=\frac{k}{1+k} .
$$

In der folgenden Tabelle 6 sind die Werte für eine Reihe von Temperaturen zusammengestellt.

Tabelle 6.

\begin{tabular}{c|r|r|r}
\hline \hline$T$ & $t$ & $100 k$ & $100 x$ \\
\hline \hline $900^{1}$ & 627 & 2.2 & 2.15 \\
$1000^{1}$ & 727 & 4.4 & 4.2 \\
$1096^{1}$ & 823 & 7.8 & 7.2 \\
1179 & 906 & 11.5 & 10.3 \\
1282 & 1009 & 17.9 & 15.2 \\
1383 & 1110 & 25.8 & 20.5 \\
1500 & 1227 & 36.6 & 26.5 \\
1600 & 1327 & 47.5 & 32.2 \\
1700 & 1427 & 59.9 & 37.5 \\
1800 & 1527 & 73.6 & 42.4
\end{tabular}

Es liegt nun nahe, zu versuchen, durch Vergleich der gefundenen Bildungswärme von Schwefelkohlenstoff, bezogen auf gasförmigen Schwefel, mit den Angaben über die Bildungswärme des Schwefelkohlenstoffs aus festem Schwefel die Verdampfungswärme des Schwefels zu berechnen.

Benutzen wir Thomsens Angaben, so ist

$$
\begin{aligned}
& \text { 1) } \mathrm{C}_{\text {amorph }}+\mathrm{S}_{2 \text { gasf. }}=\mathrm{CS}_{2 \mathrm{gasf}}+12500 \mathrm{cal} \text {, } \\
& \text { 2) } \mathrm{C}_{\text {amorph }}+2 \mathrm{~S}_{\text {fest }}=\mathrm{CS}_{2 \text { gasf. }}-26000 \mathrm{cal}^{2}
\end{aligned}
$$

Die Subtraktion 2.-1. ergibt

3) $2 S_{\text {fest }}=S_{2 \text { gasf. }}-38500$ cal.

${ }^{1}$ Die in Nernst, Experimental and theoretical Applications of Thermodynamies to Chemistry, New York 1907, S. 100, angegebenen Werte für $100 x$ sind $z \mathrm{u}$ klein. Sie sind meinen Vorversuchen entnommen, das Gleichgewicht war noch nicht erreicht, wie sich später herausstellte.

2 Thomsen, Th. U. II, S. 411. 
Nach Berthelot aber beträgt die Bildungswärme des Schwefelkohlenstoffs aus amorphem Kohlenstoff und festem Schwefel - 22100 cal. ${ }^{1}$ Bei Benutzung dieses Wertes ergibt sich

4) $2 S_{\text {fest }}=S_{2 \text { gasf. }}-34600$ cal.

Da 1) bei hoher Temperatur (ca. $1000^{\circ}$ ), 2. bei Zimmertemperatur gilt, so addiert sich auf der rechten Seite der Energiegleichung 3) und 4) noch

$$
1000\left(\mathrm{C}_{\mathrm{C}}+\mathrm{C}_{\mathrm{s}_{2}}-\mathrm{C}_{\mathrm{Cs}_{2}}\right)
$$

wo $\mathrm{C}_{\mathrm{C}}, \mathrm{C}_{\mathrm{S}_{2}}, \mathrm{C}_{\mathrm{CS}_{2}}$ die betreffenden mittleren Molekularwärmen für konstanten Druck bedeuten.

Nun ist $\mathrm{C}_{\mathrm{C}}=3.77\left(\right.$ Dewar), ${ }^{2} \mathrm{C}_{\mathrm{S}_{2}}$ lälst sich (in Analogie mit $\mathrm{O}_{2}$ ) zu etwa 8 schätzen, $\mathrm{C}_{\mathrm{CS}_{2}}$ ist nach REGNAULT ${ }^{2}=11.95$ zwischen $70^{\circ}$ und $194^{\circ}$. Bei höherer Temperatur wird es etwas wachsen. Aus diesen Molekularwärmen geht hervor, dafs der Ausdruck $\left(\mathrm{C}_{\mathrm{C}}+\mathrm{C}_{\mathrm{S}_{2}}-\mathrm{C}_{\mathrm{CS}_{2}}\right)$ von Null nicht sehr verschieden ist. Er wurde deshalb vernachlässigt.

Für den Übergang von festem Schwefel in gasförmige $S_{2}=$ Moleküle bei Zimmertemperatur und konstantem Druck finden wir also die beiden Zahlen - $38500 \mathrm{cal}$ (Tномsen) und - $34600 \mathrm{cal}$ (BerTHELOT) [ -37900 und -34000 cal für konstantes Volumen], während Preuner ${ }^{3}$ neuerdings für die gleiche Reaktion aus den Dissoziationsisothermen des Schwefels die Wärmetönung - $29400 \mathrm{cal}$ und auf anderem Wege, aus der Dissoziation des Schwefelwasserstoffs - 31100 cal berechnet (beide Zahlen beziehen sich auf konstanten Druck).

Die Bildungswärme des Schwefelkohlenstoffs ans festem Schwefel ist von Thomsen und Berthelot aus den Verbrennungswärmen ermittelt worden, ist also als kleine Differenz grofser Zahlen mit grofser Ungenauigkeit behaftet. Berücksichtigt man nur die durchschnittliche Abweichung vom Mittelwert der Einzelversuche über die Verbrennungswärme, so ergeben sich folgende Genauigkeitsgrenzen:

$$
\begin{aligned}
\left(\mathrm{C}_{\text {amorphl, }},\right. & \left.2 \mathrm{~S}_{\text {fest }}\right)-26000 \pm 1000 \mathrm{cal} \text { nach Thomsen, }{ }^{4} \\
& -22100 \pm 4300 \text { cal nach BeRtheLot. }{ }^{5}
\end{aligned}
$$

1 Berthelot, Thermochimie.

${ }^{2}$ Landolt-Börnstein-Meterhofer.

3 Zeitschr. phys. Chem. 68 (1909), 167.

${ }^{4}$ 1. c. S. 375 .

5 Ann. Chim. Phys. [3] 23, 209; [6] 22, 183; [6] 28, 138. 
Aufserdem gehen die Zahlen für die Bildungswärme von $\mathrm{CO}_{2}, \mathrm{SO}_{2}$ und $\mathrm{SO}_{3}{ }^{1}$ mit ihren Ungenauigkeiten in die Rechnung ein.

Die folgende Zusammenstellung zeigt die schwankenden Angaben über die Bildungswärmen von $\mathrm{CO}_{2}, \mathrm{SO}_{2}$ und $\mathrm{SO}_{3}$ :

$\begin{array}{lll} & \text { Thomsen: } & \text { Berthelot: } \\ \left(\mathrm{C}, \mathrm{O}_{2}\right) & 96960 \mathrm{cal} & 97300 \mathrm{cal} \\ \left(\mathrm{S}, \mathrm{O}_{2}\right) & 71080 " & 69300 " \\ \left(\mathrm{~S}, \mathrm{O}_{3}\right) & 91440 " & 91900 "\end{array}$

Hierdurch wird die Sicherheit obiger Zahlen noch weiter beeinträchtigt.

Die Angaben über die Bildungswärme des Schwefelkohlenstoffs aus festem Schwefel sind also nicht zuverlässig genug, um darauf eine genaue Berechnung der Verdampfungswärme des Schwefels zu stützen.

Der von Preuner auf zwei verschiedenen Wegen gefundene Wert hat grölsere Wahrscheinlichkeit.

\section{Das Schwefelkohlenstoffgleichgewicht und das Nernstsche Wärmetheorem.}

Die NERNsT sche Näherungsformel, angewandt auf das Schwefelkohlenstoffgleichgewicht, gewinnt folgende Gestalt:

$$
\lg k=-\frac{12500}{4.57 T}+\Sigma \nu C .
$$

In der Tat schlielst sich den Beobachtungen sehr gut eine Gleichung von dieser Form an:

$$
\lg k=-\frac{2740}{T}+1.38,
$$

wie folgende kleine Tabelle zeigt.

Tabelle 7 .

\begin{tabular}{c|c|c}
\hline \hline $\lg k$ & $T$ beob. & $T$ ber. \\
\hline \hline $0.89-2$ & 1096 & 1100 \\
$0.06-1$ & 1179 & 1180 \\
$0.25-1$ & 1282 & 1286 \\
$0.41-1$ & 1383 & 1390 \\
\hline
\end{tabular}

${ }^{1}$ Bei Berthelot wurde der Schwefelkohlenstoff zu SO $\mathrm{SO}_{3}$, bei Thomsen zu $\mathrm{SO}_{2}$ verbrannt. 
Da, wie oben gezeigt, der Einflufs der spezifischen Wärmen unerheblich zu sein scheint, so bedeutet die Konstante 1.38 die Differenz der chemischen Konstanten von $\mathrm{S}_{2}$ und $\mathrm{CS}_{2}$.

Zur Ermittelung bzw. Revision des Wertes für die chemische Konstante des Schwefelkohlenstoffs wird zurzeit im hiesigen Physikalisch-chemischen Institut die Dampfdruckkurve des Schwefelkohlenstoffs genau bestimmt. Es hat sich bereits herausgestellt, dafs der bisher angegebene Wert $3.1 \mathrm{zu}$ hoch ist. Die chemische Konstante des Schwefels dürfte um einige Zehntel gröfser als 3 sein.

Der hohe Wert bat nichts Auffälliges bei der starken Tendenz des $\mathrm{S}_{2}$-Moleküls sich zu assoziieren.

\section{Zusammenfassung der Resultate.}

1. Das Gleichgewicht der Reaktion

$$
\mathrm{C}+\mathrm{S}_{2} \text { gasf. } \rightleftharpoons \mathrm{CS}_{2} \text { gasf. }
$$

wurde in dem Temperturintervall $800-1100^{\circ}$ gemessen.

2. Aus dem gemessenen Gleichgewicht ergab sich die Bildungswärme des Schwefelkohlenstoffs, bezogen auf gasförmigen Schwefel zu 12500 cal.

3. Aus dem Vergleich dieser Zahl mit den Angaben Berthelots und Thомsens über die Bildungswärme des Schwefelkohlenstoffs aus festem Schwefel die Verdampfungs- (inkl. Dissoziations-) Wärme des Schwefels zu berechnen, ist nur annäherungsweise möglich, da diese Angaben voneinander abweichen und nicht zuverlässig genug sind.

4. Im Sinne des NeRsstschen Wärmetheorems ergibt sich aus dem Schwefelkohlenstoffgleichgewicht für die Differenz der chemischen Konstanten des $\mathrm{S}_{2}$ - und $\mathrm{CS}_{2}$-Moleküls der Wert 1.38.

Die vorstehende Arbeit wurde im Physikalisch-chemischen Institut der Universität Berlin ausgeführt. Es sei mir an dieser Stelle gestattet, meinem hochverehrten Lehrer, Herrn Prof. W. NERnsT für die Anregung zu dieser Arbeit und sein förderndes und wohlwollendes Interesse meinen verbindlichsten Dank auszusprechen. Auch Herm Prof. Bodenstein fühle ich mich für die liebenswürdige Unterstützung während seiner Tätigkeit am Berliner Institut zu grofsem Dank verpflichtet.

Berlin, Physikalisch-chemisches Institut der Universität.

Bei der Redaktion eingegangen am 25. Januar 1910. 